\title{
miR-875-5p regulates IR and inflammation via targeting TXNRD1 in gestational diabetes rats
}

\author{
SONGBO FU ${ }^{1}$, SONGQUAN FU ${ }^{2}$, XIAONI MA ${ }^{1}$, XIAOMEI YANG ${ }^{1}$ and JIZU LING ${ }^{3}$ \\ ${ }^{1}$ Department of Endocrinology, The First Hospital of Lanzhou University, Lanzhou, Gansu 730000; \\ ${ }^{2}$ Department of Respiration, The First Hospital of Lanzhou City, Lanzhou, Gansu 730050; \\ ${ }^{3}$ Department of Pediatrics, The First Hospital of Lanzhou University, Lanzhou, Gansu 730000, P.R. China
}

Received May 28, 2020; Accepted January 7, 2021

DOI: $10.3892 / \mathrm{mmr} .2021 .11942$

\begin{abstract}
Gestational diabetes mellitus (GDM) is a serious life-threatening disease that affects the mother and fetus. However, the pathogenesis of GDM is still unclear. microRNAs (miRs) play vital roles in the regulation of various cell functions. The present study aimed to investigate the effects of miR-875-5p and thioredoxin reductase 1 cytoplasmic (TXNRD1) in GDM rats and analyze the associated underlying mechanism. A GDM rat model was induced using an intraperitoneal injection of streptozotocin. miR-875-5p knockdown plasmids or TXNRD1 knockdown plasmids were injected into the rats via the caudal vein. miR-875-5p and TXNRD1 expression in the serum were detected using reverse transcription-quantitative PCR (RT-qPCR) or western blot (WB) analyses. The fasting blood-glucose (FBG), fasting serum insulin, triglyceride and high density lipoprotein levels were detected by specific commercial kits. The inflammatory response and the induction of oxidative stress were analyzed by assessing the expression of associated markers via WB, RT-qPCR or commercial kits. The pancreatic and placental injuries were detected by hematoxylin and eosin staining. The results indicated that miR-875-5p expression levels were downregulated, whereas TXNRD1 levels were upregulated in GDM rats compared with normal pregnancy rats. miR-875-5p significantly regulated TXNRD1 expression in GDM rats. miR-875-5p silencing notably reduced FBG and insulin resistance, which was accompanied by reduced expression levels of blood lipid and pro-inflammatory markers as well as reduced oxidative stress. However, the effects of miR-875-5p could be reversed by TXNRD1 silencing. Therefore, the present study indicated that miR-875-5p regulated IR and inflammation by
\end{abstract}

Correspondence to: Dr Songbo Fu, Department of Endocrinology, The First Hospital of Lanzhou University, 1 Donggangxi Road, Chengguan, Lanzhou, Gansu 730000, P.R. China

E-mail: songbofu123@163.com

Key words: gestational diabetes mellitus, microRNA-875-5p, thioredoxin reductase 1 cytoplasmic, inflammation targeting TXNRD1 in GDM rats. miR-875-5p and TXNRD1 may be considered as potential targets for treating GDM.

\section{Introduction}

Gestational diabetes mellitus (GDM) is one of the common complications noted in the perioperative period, which affects normal glucose metabolism before pregnancy (1). Different degrees of abnormal glucose tolerance and elevated fasting blood glucose (FBG) have been reported during pregnancy. GDM is a serious life-threatening disease affecting the mother and fetus and its incidence is increasing every year (2) in China. High blood glucose (BG) may increase the incidence of premature delivery and miscarriage. However, the pathogenesis of GDM is still unclear (2). The majority of previous studies have demonstrated that insulin resistance (IR) is one of the key factors involved in the pathogenesis of GDM. In addition, inflammatory factors and oxidative stress also play an important role in this process $(3,4)$. Several studies have reported that the expression levels of antioxidant genes thioredoxin reductase 1 cytoplasmic (TXNRD1) and superoxide dismutase (SOD) are upregulated in type 2 diabetes $(5,6)$.

Moreover, a direct link between GDM and persistent inflammation has been reported in pregnant women (7). Previous studies have also demonstrated that GDM induces abnormal microRNA (miRNA/miR) expression $(8,9)$. miRNAs can be used as diagnostic tools for GDM and may affect the regulation of several cell functions (10). The expression levels of miR-875-5p have been demonstrated to be downregulated in patients with GDM (11). It has also been revealed that miR-875-5p can be used as a biomarker for GDM, suggesting its vital role in the diagnosis of this disease (11). It has also been illustrated that miR-876-5p is involved in the development of cancer and that it can be used for the development of potential novel anticancer therapies $(12,13)$. TXNRD1 gene encodes thioredoxin reductase, and is also known as the TrxR1 gene. TrxR1 is a member of the thioredoxin system, which plays an important role in redox regulation and antioxidant defense by reducing Trx levels in order to maintain the reduction state of intracellular proteins (14). TXNRD1 is predominantly present in the cytoplasm. Cells can fight oxidative stress by upregulating TXNRD1 levels to eliminate hydrogen peroxide and inhibit apoptosis. A previous study indicated that the 
increase in TXNRD1 levels could play a significant role in treating patients with uncontrolled diabetes $(15,16)$. The TrxR/Trx system plays an important role in the inhibition of oxidative stress, reduction of apoptosis (17), DNA synthesis and the regulation of the NF- $\mathrm{KB}$ signaling pathway $(18,19)$. A close association has been demonstrated between the inflammatory response and TXNRD1 (20). Inflammation can regulate TXNRD1 production (21). Therefore, the present study aimed to assess the mechanism of action of miR-875-5p and TXNRD1 in GDM rats.

\section{Materials and methods}

Animals. A total of 200 Wistar rats (weight, 180-270; age, 8 weeks; female, 100, male, 100) were purchased from Junke Biological Co., Ltd. The female rats were fed with normal feed (control group, $\mathrm{n}=10$ rats) or a high fat and high sucrose diet (GDM group, $\mathrm{n}=90$ rats) for 8 weeks at $23-25^{\circ} \mathrm{C}$ with a relative humidity of $65-70 \%$ in a $12 \mathrm{~h}$ light/dark cycle. Subsequently, female rats that were in proestrus were caged with males overnight. The rats were identified as pregnant (day 0 ) according to the observed sperm or mucus plug by microscopy the following day. A total of 100 female rats were identified to be pregnant. The rats in the GDM group were injected intraperitoneally with streptozotocin ( $35 \mathrm{mg} / \mathrm{kg}$, Sigma-Aldrich; Merck KGaA) to induce gestational diabetes. If the FBG level was stable at $13.5 \mathrm{mmol} / \mathrm{l}$ following 24 and $72 \mathrm{~h}$, the GDM rat model was considered to have been successfully established (22). GDM rats were randomly divided into the following groups ( $n=10$ per group): i) GDM group, no treatment; ii) GDM+vector group, 4 days following induction, rats were injected with miR-875-5p inhibitor negative control (NC, $10 \mu \mathrm{M}$ vector) every 3 days via the tail vein; and iii) GDM+miR-875-5p inhibitor group, 4 days following induction, rats were injected with miR-875-5p inhibitor ( $10 \mu \mathrm{M}$ vector) every 3 days via the tail vein. To further investigate the molecular mechanism of miR-875-5p and TXNRD1, the remaining GDM rats were randomly divided into five groups ( $\mathrm{n}=10$ per group): i) GDM, no treatment; ii) GDM+sh-NC; iii) GDM+sh-TXNRD1;iv) GDM+miR-875-5p inhibitor+sh-NC (100 $\mu \mathrm{g}$ pGPU6/GFP/Neo-shRNA-NC); and v) GDM+miR-875-5p inhibitor+sh-TXNRD1 (100 $\mu \mathrm{g}$ pGPU6/GFP/Neo-shTXNRD1). The sequences used for the inhibition of miR-875-5p or TXNRD1 were asfollows: miR-875 inhibitor,5'-CACCUGAUAAAACUG AGGUAUA-3'; inhibitor NC, 5'-AUCGACAGGGUUAACUC CACGA-3'; sh-TXNRD1 forward, 5'-CACCGCAATGATCT TGAGTTCTATGCGAACATAGAACTCAAGATCATTGC-3' and reverse, 5'-AAAAGCAATGATCTTGAGTTCTATGTTC GCATAGAACTCAAGATCATTGC-3'; shRNA-NC forward, 5'-CACCGACATACTCTGTTAGTGTAGTCGAAACTACAC TAACAGAGTATGTC-3' and reverse, 5'-AAAAGACATA CTCTGTTAGTGTAGTTTCGCACTACACTAACAGAGTA TGTC-3'. All sequences were obtained from Guangzhou RiboBio Co., Ltd.

After 7 days, the rats were anesthetized by intraperitoneal injection of $1 \%$ pentobarbital sodium $(50 \mathrm{mg} / \mathrm{kg})$, followed by exsanguination $10 \mathrm{~h}$ after fasting. After anesthesia, blood ( $5 \mathrm{ml})$ was collected from the abdominal aorta for subsequent experiments. Blood, pancreatic islets and placental tissues of the rats were collected. After the blood samples were collected, they were stored overnight in the refrigerator at $4^{\circ} \mathrm{C}$. Subsequently, the serum was collected by centrifugation at $4^{\circ} \mathrm{C}$ at $1,409 \times \mathrm{g}$ for $15 \mathrm{~min}$ and divided into $100 \mu \mathrm{l}$ samples, which were stored at $-80^{\circ} \mathrm{C}$. Other tissues and organs were separated and stored at $-80^{\circ} \mathrm{C}$ or fixed in $4 \%$ formaldehyde solution at room temperature for $48 \mathrm{~h}$. The experimental process is presented in Fig. S1. All experimental procedures were approved by the Animal Experimental Ethics Committee of The First Hospital of Lanzhou University (approval no. LDYYLL2020-207).

Reverse transcription-quantitative PCR (RT-qPCR). Total RNA was extracted using the TRIzol ${ }^{\circledR}$ reagent (Invitrogen; Thermo Fisher Scientific, Inc.), according to the manufacturer's protocol, and reverse transcribed into cDNA using miScript II RT Kit (Shanghai Beinuo Biotechnology Co., Ltd.) at $37^{\circ} \mathrm{C}$ for $20 \mathrm{~min}$. miRNA was amplified using the miScript SYBR Green PCR Kit (Shanghai Beinuo Biotechnology Co., Ltd.). The other part of collected serum or pancreatic islet tissues was used to reverse transcribe into cDNA with the PrimeScript RT Reagent Kit (Takara Bio, Inc.) according to the manufacturer's protocol. mRNA was amplified using the SYBR Premix ExTaq (Takara Bio, Inc.). The thermocycling conditions were performed for qPCR as follows: Initial denaturation at $95^{\circ} \mathrm{C}$ for $3 \mathrm{~min}$, followed by 45 cycles of $95^{\circ} \mathrm{C}$ for $5 \mathrm{sec}, 60^{\circ} \mathrm{C}$ for $20 \mathrm{sec}$ and $72^{\circ} \mathrm{C}$ for $15 \mathrm{sec}$. The $\mathrm{Cq}$ value of the target gene of each sample was normalized against U6 or GADPH, and the relative expression levels were measured using the $2^{-\triangle \Delta C q}$ method (23). The primer sequences used were as follows: miR-875-5p forward, 5'-TATACCTCAGTTTTATC AGGTG-3' and reverse, 5'-GCGGCCGCGTGCATAGCTTCT GTAAAGG-3'; TXNRD1 forward, 5'-CTTCCACGTACTGG GTCCAAATG-3' and reverse, 5'-TCACCGACAGCGTTGT AAATATCTC-3'; CRP forward, 5'-CATCTGTGCCACCTGG GAGTC-3' and reverse, AAGCCACCGCCATACGAGTC-3'; TNF- $\alpha$ forward, 5'-TGAGCACAGAAAGCATGATC-3' and reverse, 5'-CATCTGCTGGTACCACCAGTT-3'; IL-6 forward, 5'-GGCCCTTGCTTTCTCTTCG-3' and reverse, 5'-ATAATA AAGTTTTGATTATGT-3'; U6 forward, 5'-CTCGCTTCGGC AGCACA-3' and reverse, 5'-AACGCTTCACGAATTTG CGT-3'; and GADPH forward, 5'-TCACCATCTTCCA GGAGCGA-3' and reverse, 5'- ACGCCAGTAGACTCCACG ACA-3'.

Detection of FBG, serum fasting insulin (FINS), homeostatic model assessment of insulin resistance (HOMA-IR), total cholesterol (TC), triglyceride (TG) and high-density lipoprotein (HDL) levels. Following fasting for $10 \mathrm{~h}$, the blood samples of the rats in each group were collected from the abdominal aorta $(5 \mathrm{ml})$. The blood samples were placed on blood glucose test strips to record FBG levels (Nova Biomedical Corporation). The detection of FINS was performed by enzyme linked immunosorbent assay (cat. no. hzA448Ra; Shanghai Huzhen Biological Technology Co., Ltd.). The HOMA-IR was calculated using the following formula: [FBG (mmol/l) x FINS (mU/l)] / 22.5. TG and HDL levels were determined by an BK-400 automatic biochemical analyzer (BioBase).

Western blot analysis. Total protein was isolated from pancreatic islet tissue using RIPA lysis buffer (Sigma-Aldrich; Merck KGaA). The concentration of total protein of the 
pancreatic islet tissue was determined using the Bradford method. Proteins $(50 \mu \mathrm{g})$ were separated via SDS-PAGE on a $10 \%$ gel, and then transferred to PVDF membranes, which were blocked using $5 \%$ skimmed milk powder for $1 \mathrm{~h}$ at $4^{\circ} \mathrm{C}$. The membranes were incubated with the following primary antibodies for $2 \mathrm{~h}$ at room temperature: Anti-C reactive protein (CRP; cat. no. ab207756; 1:1,000), TNF- $\alpha$ (cat. no. ab205587; 1:1,000), IL-6 (cat. no. ab208113; 1:1,000), TXNRD1 (cat. no. ab124954; 1:1,000) and GAPDH (cat. no. ab9485; 1:1,000) (all purchased from Abcam). Subsequently, membranes were incubated with the HRP-conjugated secondary antibody (cat. no. ab7090; 1:10,000; Abcam) for $1 \mathrm{~h}$ at room temperature. Chemiluminescence enhancement reagent (Thermo Fisher Scientific, Inc.) was added to react with the protein band at room temperature for $1 \mathrm{~min}$. The results were analyzed using the ImageJ software (version 1.46r; National Institutes of Health).

Oxidative stress. Following blood collection, the pancreatic tissues were removed, cut into pieces and further homogenized. Following centrifugation at $1,917 \times \mathrm{g}$ for $5 \mathrm{~min}$ at $4^{\circ} \mathrm{C}$, the supernatant was obtained. The activity of SOD and catalase (CAT), and the content of malondialdehyde (MDA) in the pancreatic tissues were determined by UV-visible spectrophotometry using assay kits (SOD, cat. no. S0086; CAT, cat. no. S0082; MDA, cat. no. S0131S; all purchased from Beyotime Institute of Biotechnology), according to the manufacturer's instructions. The OD value of each group was detected at 560, 405 or $532 \mathrm{~nm}$ for SOD activity, CAT activity and MDA content, respectively.

Hematoxylin and eosin $(H \& E)$ staining. Following anesthesia of the animals with $1 \%$ sodium pentobarbital $(50 \mathrm{mg} / \mathrm{kg}$, abdominal injection), pancreatic and placental tissues were obtained. The tissues were fixed in $4 \%$ formaldehyde solution at room temperature for $48 \mathrm{~h}$, embedded in paraffin (thickness of $5 \mu \mathrm{m}$ ) and stained with H\&E. Hematoxylin staining was performed for $9 \mathrm{~min}$, followed by eosin staining for $2 \mathrm{~min}$. The pathophysiological changes of the pancreatic and placental tissues were observed by light microscopy (magnification, x200).

Cell lines and luciferase reporter assay. INS-1 cells (American Type Culture Collection) were cultured in RPMI-1640 medium (containing $11.1 \mathrm{mM}$ glucose; Thermo Fisher Scientific, Inc.), in the presence of $10 \%$ fetal bovine serum (Gibco; Thermo Fisher Scientific, Inc.), $0.11 \mathrm{~g} / 1$ sodium pyruvate, $50 \mu \mathrm{M} \beta$-mercaptoethanol, and $1 \%$ penicillin-streptomycin. The cells were maintained at $37^{\circ} \mathrm{C}$ with $5 \% \mathrm{CO}_{2}$. Cells were seeded into 96-well plates at a density of 40-60\% and cultured in RPMI-1640 medium without penicillin-streptomycin for $24 \mathrm{~h}$, and subsequently transfected with specific plasmids. miR-875-5p mimic (5'-UAUACCUCAGUUUUAUCAG GUG-3'; 50 nM; Guangzhou RiboBio Co., Ltd.) or scrambled miR-875-5p (5'-AUGCAAGGUCGGGGUAACCUCC-3'; $50 \mathrm{nM}$; Guangzhou RiboBio Co., Ltd.) were transfected into INS-1 cells with Lipofectamine ${ }^{\circledR} 2000$ for $48 \mathrm{~h}$ at $37^{\circ} \mathrm{C}$ (Invitrogen; Thermo Fisher Scientific, Inc.). Then, cells were collected and the expression of miR-875-5p was determined using RT-qPCR. The relationship between miR-875-5p and the 3 ' untranslated region (UTR) of TXNRD1 was predicted using Starbase (starbase.sysu.edu.cn/). The interaction was further confirmed by performing dual-luciferase reporter assays. Mutant (MUT) 3'UTR of TRXR1 dual-luciferase reporter vectors (Zeye Bio-Web) were constructed using a Site-directed Mutagenesis Kit (cat. no. B639281; Sangon Biotech Co., Ltd.). Wild-type (WT) or MUT vectors and miR-875-5p mimic or scrambled miR-875-5p were co-transfected into the cells using Lipofectamine ${ }^{\circledR} 2000$ (Invitrogen; Thermo Fisher Scientific, Inc.). At $48 \mathrm{~h}$ post-transfection, luciferase activities were detected using a Luciferase Reporter Assay Kit (BioVision, Inc.) and a GloMax ${ }^{\circledR}$ Discover Microplate Reader (Promega Corporation). Firefly luciferase activities were normalized to Renilla luciferase activities.

Statistical analysis. Data were analyzed using GraphPad Prism software (version 8; GraphPad Software, Inc.). The data are presented as the mean \pm SD. The comparisons among different groups were performed using ANOVA followed by Tukey's post hoc test. The unpaired Student's test was performed between two groups. $\mathrm{P}<0.05$ was considered to indicate a statistically significant difference.

\section{Results}

miR-875-5p regulates $F B G, I R$ and blood lipid levels in GDM rats. The miR-875-5p expression levels in GDM rats were initially analyzed by RT-qPCR. The results indicated that the serum miR-875-5p levels of GDM rats were significantly decreased compared with those of the control group (Fig. 1A). Subsequently, the functions of miR-875-5p were assessed through a series of experiments. miR-875-5p knockdown vectors were injected into GDM rats through the tail vein. Serum miR-875-5p levels were significantly reduced following transfection with the miR-875-5p inhibitor (Fig. 1B). Furthermore, the miR-875-5p inhibitor significantly decreased FBG and FINS levels in GDM rats compared with those in the GDM group (Fig. 1C). In addition, GDM-induced TC and TG levels were significantly decreased, whereas the level of HDL was increased in the miR-875-5p inhibitor group compared with the GDM group (Fig. 1D). The results implied that decreased miR-875-5p expression levels played protective roles in reducing FBG, IR and blood lipid levels in GDM rats.

miR-875-5p inhibitor reduces inflammation in the pancreatic islet tissues of GDM rats. CRP is a non-specific sensitive indicator of the inflammatory response that is closely associated with IR (24). CRP can inhibit the activity of the insulin receptor tyrosine kinase and induce the phosphorylation reaction of insulin receptor substrates (25). Therefore, the insulin synthesis and secretory mechanism are disrupted, which eventually lead to IR. TNF- $\alpha$ can enhance IR by directly inhibiting the expression of the glucose transporter T4 in adipocytes. IL-6 can reduce the sensitivity of the body to insulin and promote the secretion of growth hormones and glucocorticoids, which result in increased IR and BG levels $(26,27)$. Therefore, the present study analyzed the expression levels of CRP, TNF- $\alpha$ and IL- 6 in the pancreatic islet tissues in order to assess the effects of miR-875-5p on inflammation. The results indicated that miR-875-5p inhibition significantly reduced CRP, TNF- $\alpha$ and IL-6 expression levels in GDM rats (Fig. 2A and B). The induction of oxidative stress in the pancreatic tissues was 

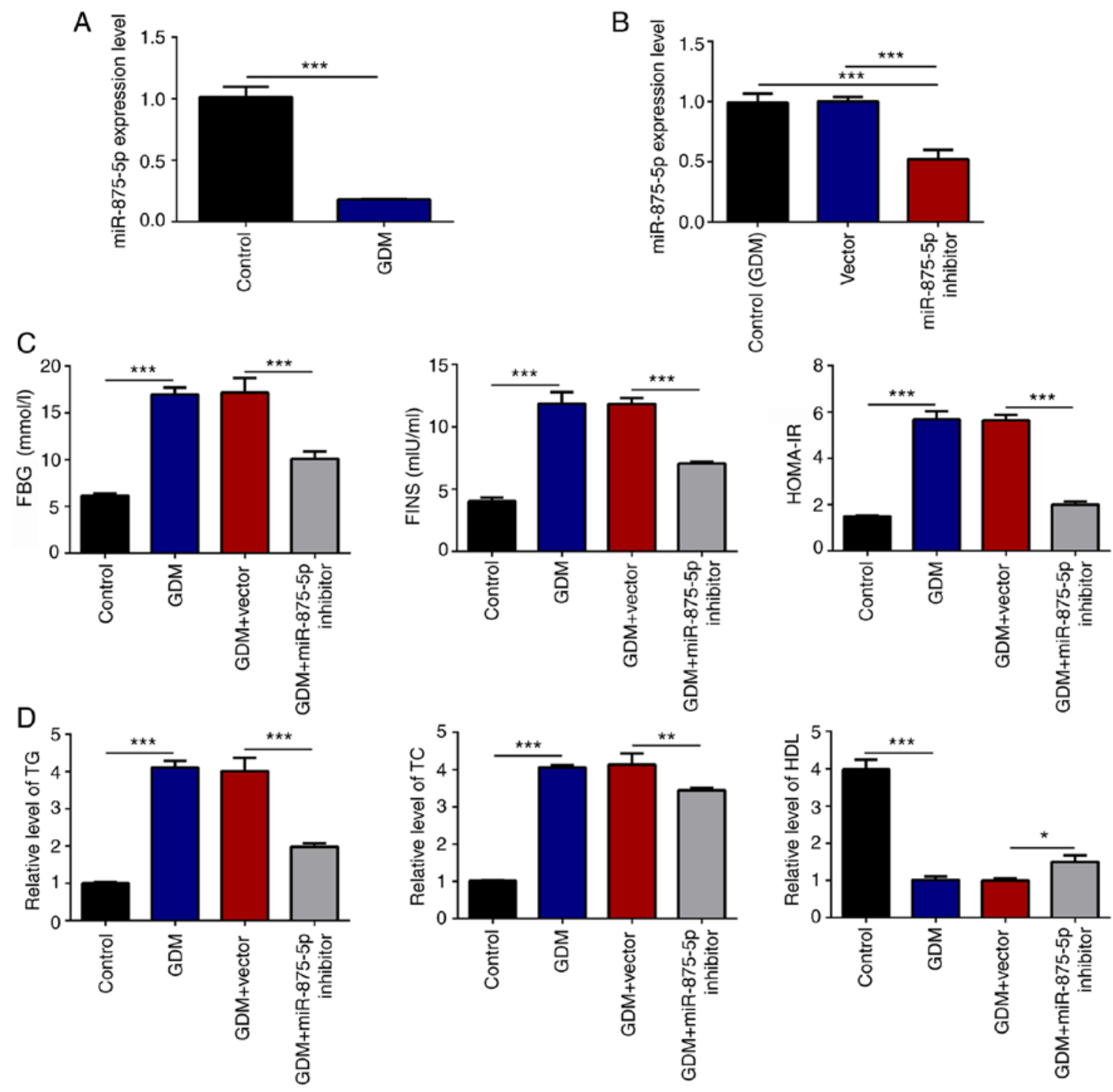

Figure 1. miR-875-5p knockdown reduces FBG, IR and blood lipid levels in GDM rats. (A) miR-875-5p levels in serum were significantly reduced in GDM rats, as determined by RT-qPCR. (B) Transfection with miR-875-5p inhibitor significantly reduced miR-875-5p expression in the serum of rats, as analyzed by RT-qPCR. (C) miR-875-5p inhibitor significantly reduced FBG, FINS and HOMA-IR blood levels. (D) Blood lipids levels were significantly affected by transfection with the miR-875-5p inhibitor. $\mathrm{n}=10$, data are presented as the mean $\pm \mathrm{SD}$. ${ }^{*} \mathrm{P}<0.05,{ }^{* *} \mathrm{P}<0.01,{ }^{* * *} \mathrm{P}<0.001$. miR, microRNA; GDM, gestational diabetes mellitus; RT-qPCR, reverse transcription-quantitative PCR; FBG, fasting blood glucose; FINS, serum fasting insulin; HOMA-IR, homeostatic model assessment of insulin resistance; TG, triglyceride; TC, total cholesterol; HDL, high-density lipoprotein.

evaluated by analysis of SOD, CAT and MDA levels (Fig. 2C). The results indicated that the enzyme activity of SOD was significantly increased following suppression of miR-875-5p levels in GDM rats compared with that of the GDM group (Fig. 2C). The levels of the anti-oxidative stress marker CAT were also significantly increased, whereas the concentration of MDA was decreased.

miR-875-5p inhibitor alleviates the pathological damage on the pancreatic and placental tissues of GDM rats. The pancreatic tissue presented normal morphology in the control group, whereas in the GDM group it demonstrated notable pathological changes, accompanied by varying degrees of inflammatory cell infiltration. The islets were markedly atrophic, with a reduced number of cells, sparse distribution and disordered arrangement. Following treatment of the animals with the miR-875-5p inhibitor, the pancreatic tissue lesions were notably decreased (Fig. 3A). In the control group, maturation of placental cell differentiation was noted, whereas the distribution was uniform and dense and the size of intercellular space was uniform and regular. The capillary blood vessels were evenly distributed and a large number of red blood cells were observed in the blood vessels. The cells in the placenta of the rats in the GDM group (Fig. 3B) were loosely distributed and disordered and the intercellular space was increased. Capillary distribution was reduced and the presence of red blood cells was rare. However, miR-875-5p inhibitor treatment markedly reduced placental injury in GDM rats.

miR-875-5p regulates the expression levels of TXNRD1. Subsequently, the effects of miR-875-5p on TXNRD1 expression were evaluated. First, the expression of miR-875-5p was determined in INS-1 cells following transfection with a miR-875-5p mimic. A significant increase in the expression 

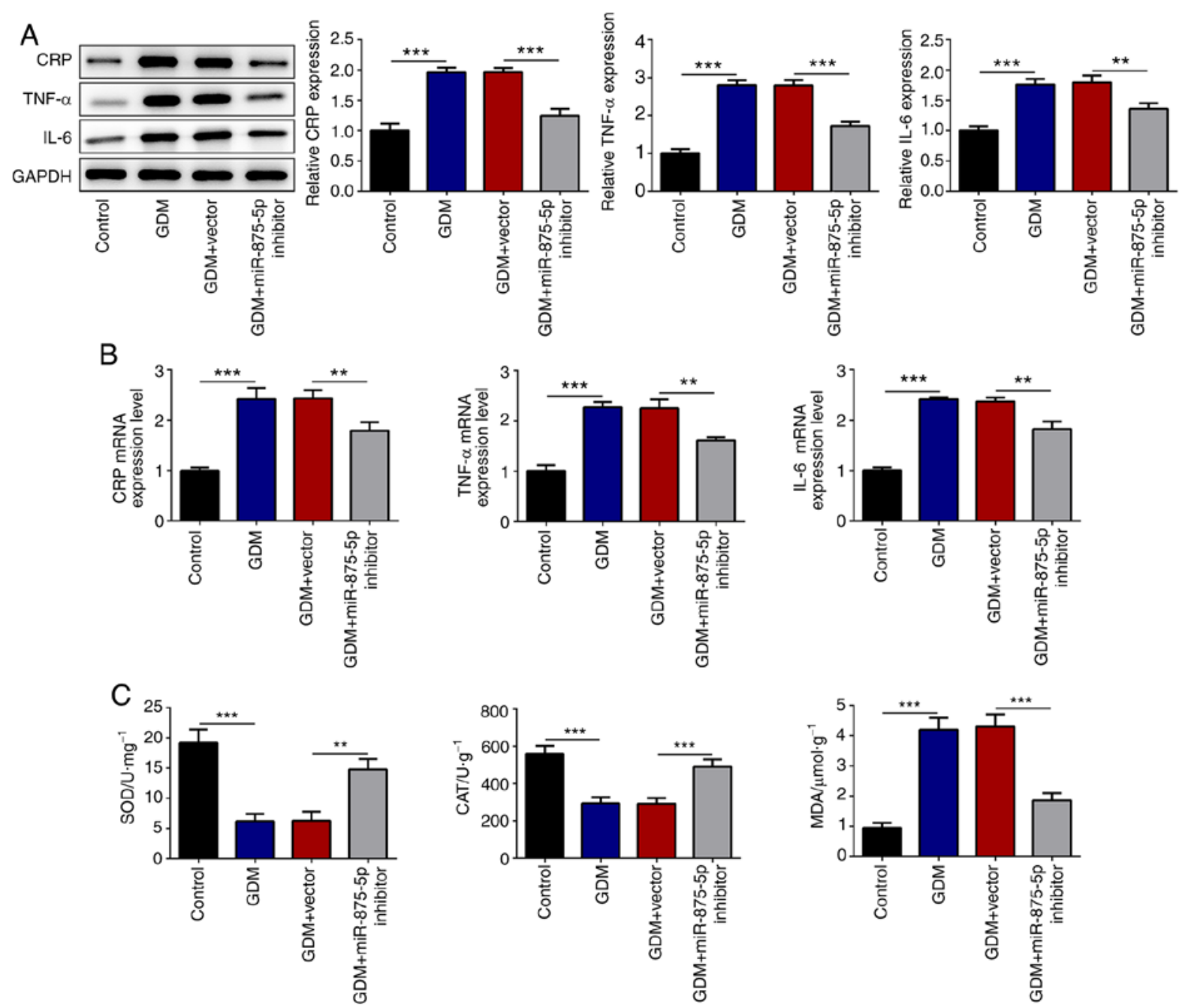

Figure 2. miR-875-5p inhibitor significantly reduces the levels of inflammatory markers and alters the levels of oxidative stress mediator. (A) Transfection with miR-875-5p inhibitor significantly reduced the protein expression of pro-inflammatory markers in the pancreatic islet tissues, as determined by western blotting. (B) Transfection with miR-875-5p inhibitor significantly reduced the mRNA expression of pro-inflammatory markers in the pancreatic islet tissues, as determined by reverse transcription-quantitative PCR. (C) Levels of oxidative stress markers were significantly altered following transfection with miR-875-5p inhibitor. $\mathrm{n}=10$, data are presented as the mean $\pm \mathrm{SD} .{ }^{* *} \mathrm{P}<0.01,{ }^{* * * *} \mathrm{P}<0.001$. miR, microRNA; GDM, gestational diabetes mellitus; CRP, C reactive protein; SOD, superoxide dismutase; CAT, catalase; MDA, malondialdehyde.

A

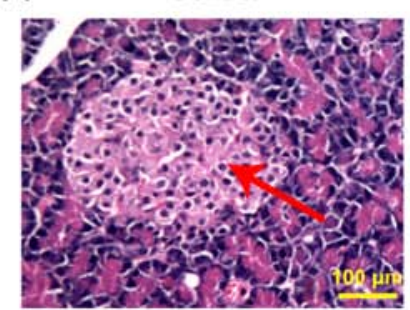

GDM+vector

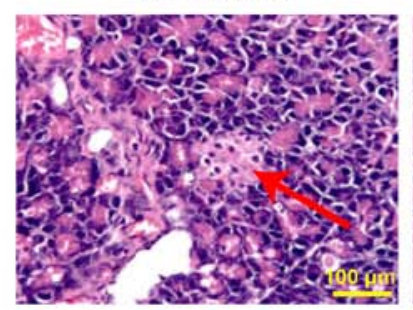

GDM

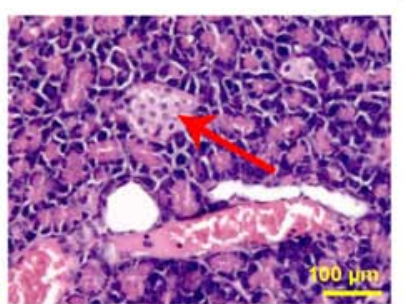

GDM+miR-875-5p inhibitor

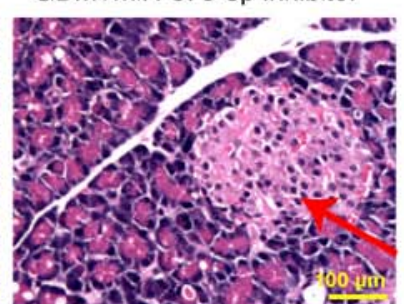

B

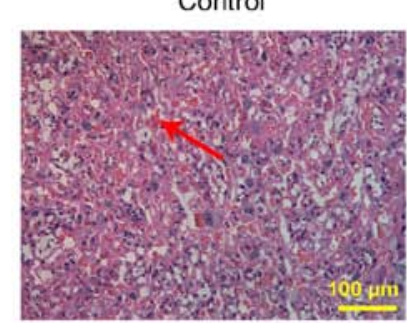

$$
\text { GDM+vector }
$$

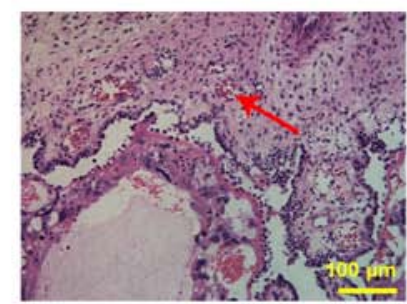

GDM

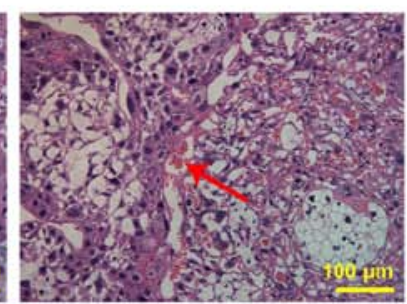

GDM+miR-875-5p inhibitor

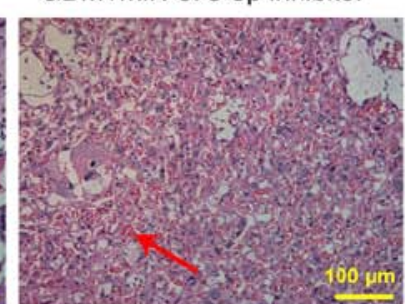

Figure 3. miR-875-5p knockdown ameliorates pancreas and placenta injury. (A) Transfection with miR-875-5p inhibitor notably improved pancreatic tissue injury in GDM rats. Arrows indicate islet cells. (B) Transfection with miR-875-5p inhibitor notably improved placental tissue injury in GDM rats. Arrows indicate blood vessels and red blood cells. $n=10$. miR, microRNA; GDM, gestational diabetes mellitus. 


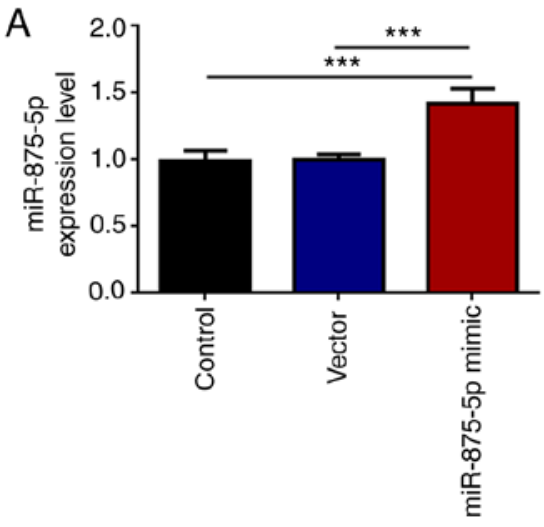

C TXNRD1 MUT : 5' CAUUUUUAUAAACUCCAUU 3'
TXNRD1 WT : 5' CAUUAUUAUAAAGAGGUAU 3'
B

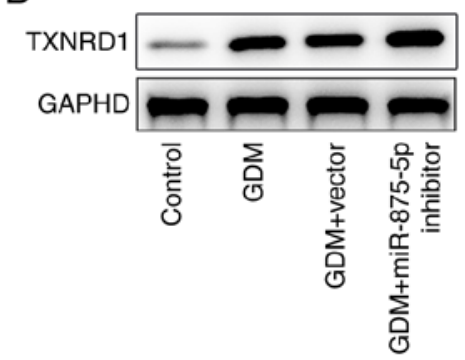

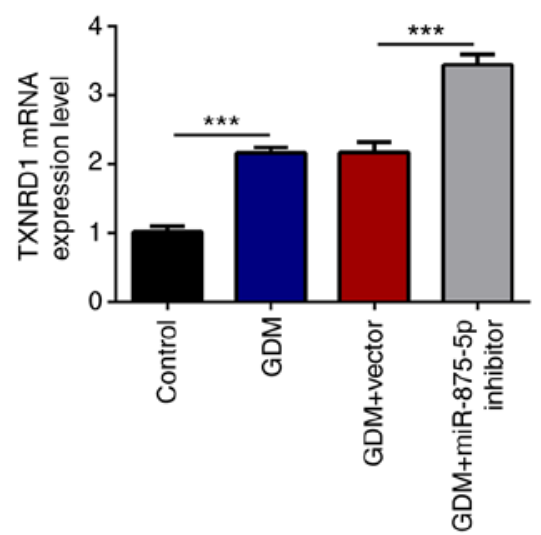

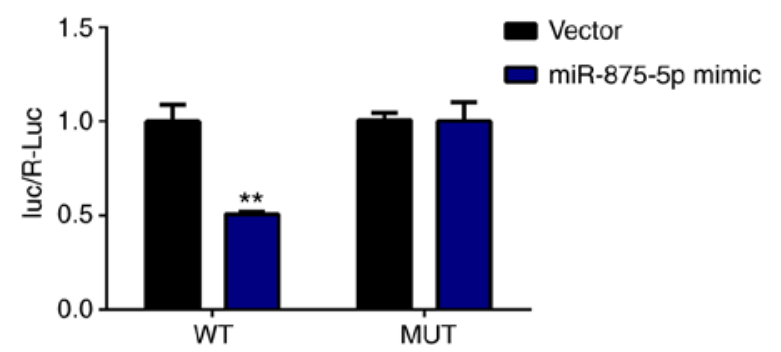

Figure 4. miR-875-5p knockdown upregulates the transcription and translation levels of TXNRD1. (A) Following transfection of INS-1 cells with miR-875-5p mimic, miR-875-5p expression significantly increased. (B) Transfection with miR-875-5p inhibitor significantly increased the TXNRD1 expression in the serum of GDM rats. (C) StarBase was used to predict that miR-875-5p can bind to the 3' untranslated region of TXNRD1, and the luciferase activity was significantly reduced by transfection with the miR-875-5p mimic in the WT group. $\mathrm{n}=10$, data are presented as the mean $\pm \mathrm{SD}$. ${ }^{* *} \mathrm{P}<0.01,{ }^{* * * *} \mathrm{P}<0.001$ vs. miR-875-5p-NC. miR, microRNA; GDM, gestational diabetes mellitus; TXNRD1, thioredoxin reductase 1 cytoplasmic; WT, wild-type; MUT, mutant; $\mathrm{NC}$, negative control.

of miR-875-5p was observed in INS-1 cells with miR-875-5p mimic (Fig. 4A). Compared with the GDM+vector group, miR-875-5p inhibitor significantly upregulated the levels of TXNRD1 in the serum of GDM rats (Fig. 4B), indicating that miR-875-5p could regulate the expression of TXNRD1 in GDM rats. The StarBase database predicted that miR-875-5p could bind to the 3'UTR of TXNRD1, which was further confirmed using a dual-luciferase reporter gene assay. The luciferase activity in the TXNRD1 WT vector co-transfected with miR-875-5p mimic was significantly reduced compared with that of the vector group, whereas no differences were observed in the TXNRD1 MUT vectors co-transfected with miR-875-5p mimic or scrambled miR-875-5p (Fig. 4C).

TXNRD1 inhibitor reverses the effects of miR-875-5p on GDM rats. Cells were transfected with sh-TXNRD1, which significantly suppressed the expression of TXNRD1 in the serum of GDM rats (Fig. 5A and B). Transfection with sh-TXNRD1 significantly reversed the effects of miR-875-5p inhibitor on glucose, FINS and HOMA-IR (Fig. 5C), inflammatory indicators (CRP, TNF- $\alpha$ and IL-6; Fig. 5D and E), and oxidative stress levels (SOD, CAT and MDA; Fig. 5F). In addition, TXNRD1 knockdown markedly altered the effects of miR-875-5p inhibitor on pancreatic and placental injury in GDM rats (Fig. 6A and B).

\section{Discussion}

GDM is a serious life-threatening disease affecting the mother and fetus that displays an increasing incidence annually in China (2). High BG contributes to an increase in the incidence of premature delivery and abortion (28). The present study indicated that transfection with miR-875-5p inhibitor played protective effects by reducing FBG, IR and blood lipid levels, along with improved pancreatic and placental injury in GDM rats. In addition, the levels of the pro-inflammatory and oxidative stress markers were significantly reduced, which implied that the miR-875-5p inhibitor could reduce inflammation in pancreatic islets and oxidative stress in the liver of GDM rats. The data indicated that the effects of miR-875-5p inhibitor could be significantly reversed by TXNRD1 inhibition. Therefore, TXNRD1 played a protective role in GDM rats. Due to the low expression of miR-875-5p noted in the plasma of GDM rats under normal physiological conditions, the expression of TXNRD1 may be maintained at a relatively low level. However, in GDM, the decreased expression of miR-875-5p alleviates its inhibition to TXNRD1 mRNA, which may be one of the underlying mechanisms for the increased expression of reactive TXNRD1 to exert antioxidation.

TrxR has been demonstrated to play a key role in redox regulation and antioxidant protection and is considered a potential target in managing metabolic syndromes (29). IR is closely associated with reduced oxidative stress in GMD. Oxidative stress is considered to be involved in the induction of IR (30-32). Therefore, TrxR1 can decrease IR by suppressing oxidative stress. TrxR is also involved in the regulation of inflammatory factors, cell proliferation, apoptosis and embryonic development (33-36). The results indicated that the expression levels of TRXR1 mRNA and protein were 


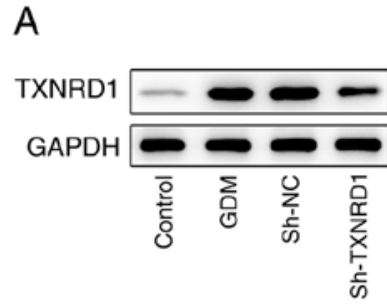

C

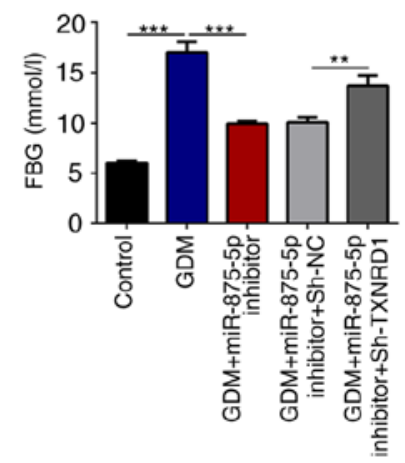

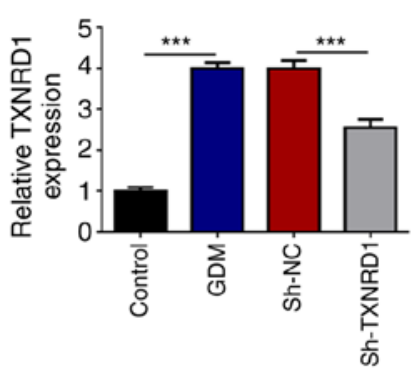
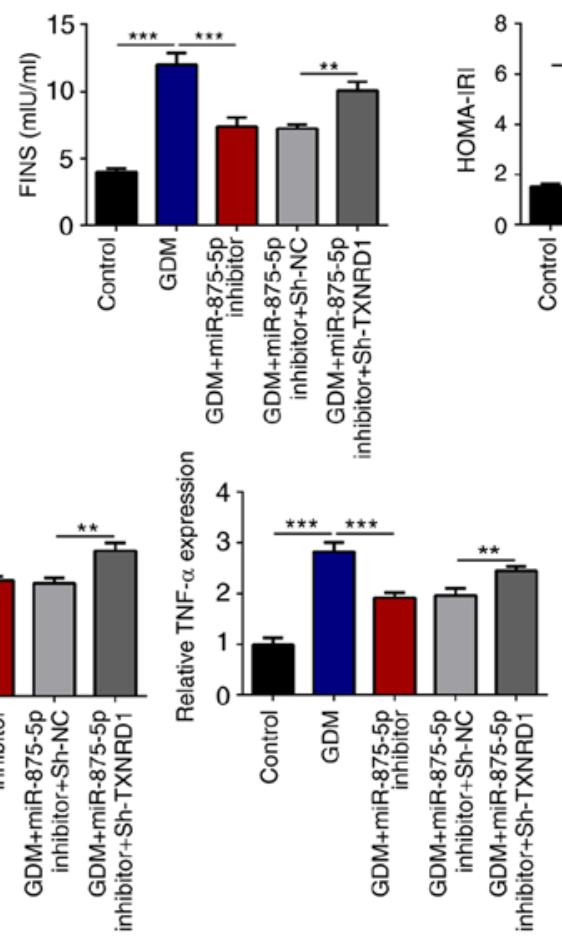

$\mathrm{B}$
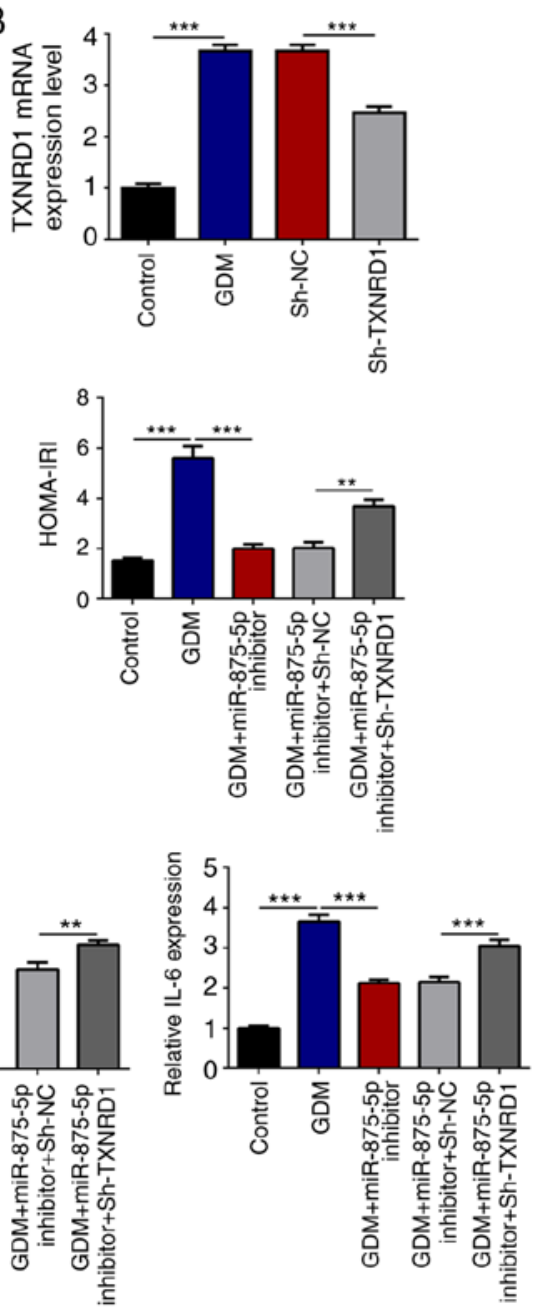

E
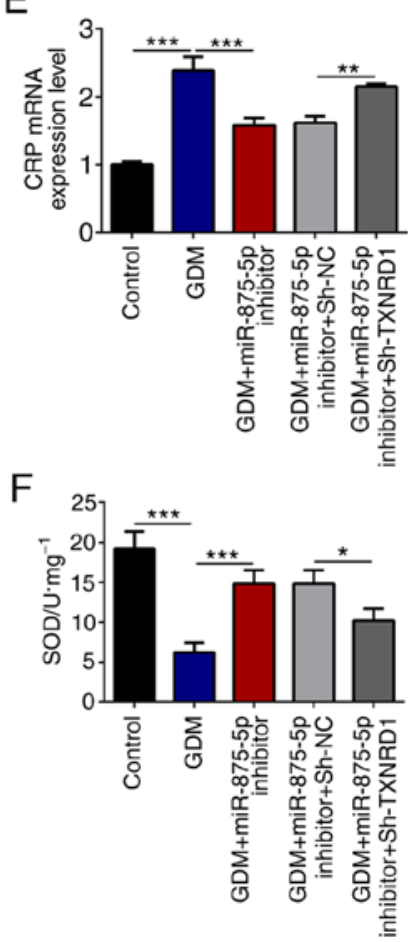
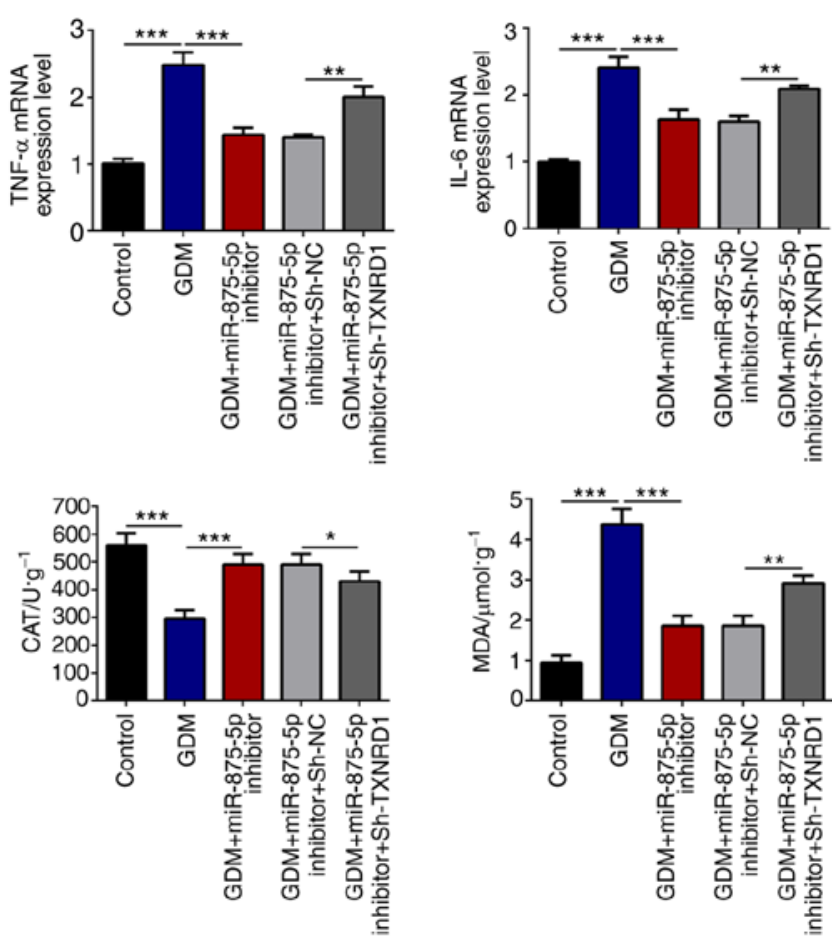

Figure 5. TXNRD1 knockdown reverses the effects of miR-875-5p inhibitor. (A and B) Transfection with sh-TXNRD1 suppressed the expression of TXNRD1 in the serum of GDM rats. (C) TXNRD1 knockdown increased FBG and IR in the blood of GDM rats. (D and E) TXNRD1 knockdown increased expression levels of pro-inflammatory markers in the pancreatic islet tissues. (F) Oxidative stress levels in the pancreatic islet tissues were altered by TXNRD1 knockdown. $\mathrm{n}=10$, data are presented as the mean $\pm \mathrm{SD} .{ }^{*} \mathrm{P}<0.05,{ }^{* * *} \mathrm{P}<0.01,{ }^{* * *} \mathrm{P}<0.001$. miR, microRNA; GDM, gestational diabetes mellitus; sh-, short hairpin RNA; TXNRD1, thioredoxin reductase 1 cytoplasmic; FBG, fasting blood glucose; FINS, serum fasting insulin; HOMA-IR, homeostatic model assessment of insulin resistance; CRP, C reactive protein; SOD, superoxide dismutase; CAT, catalase; MDA, malondialdehyde; NC, negative control. 
A

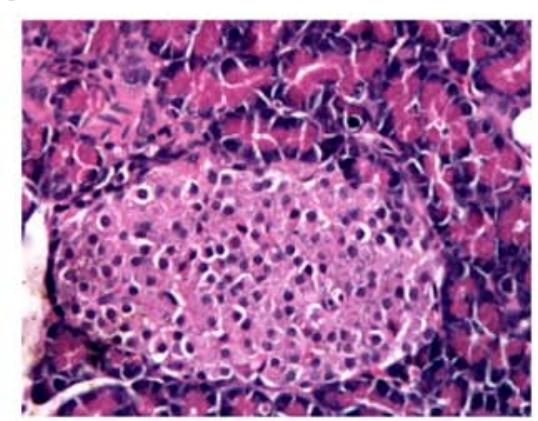

GDM+miR-875-5p inhibitor+Sh-NC

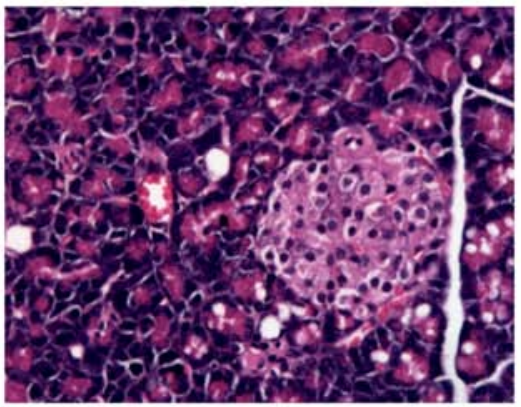

B

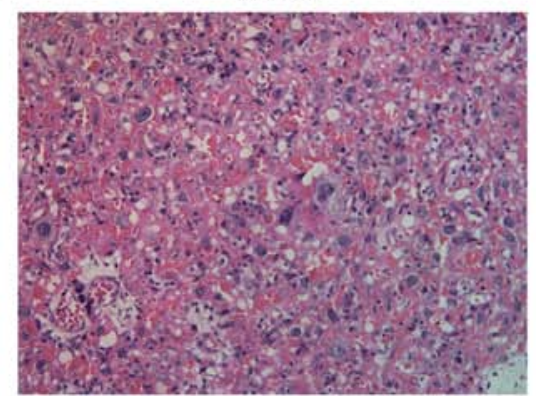

GDM+miR-875-5p inhibitor+Sh-NC

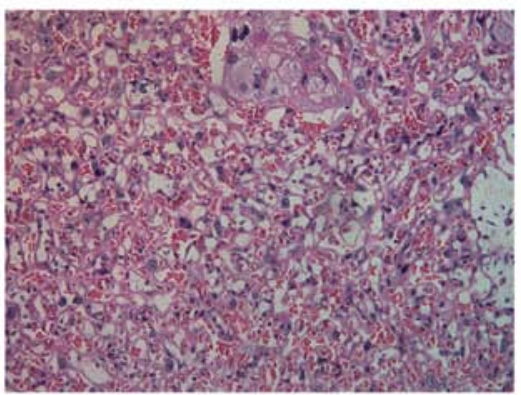

GDM

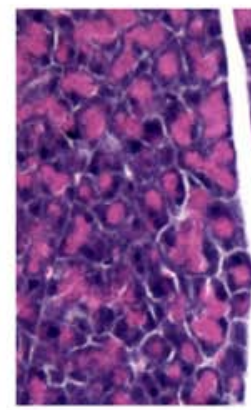

GDM+miR-875-5p inhibitor+Sh-TXNRD1

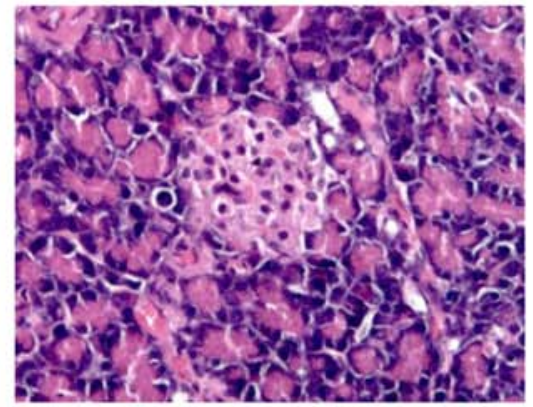

GDM

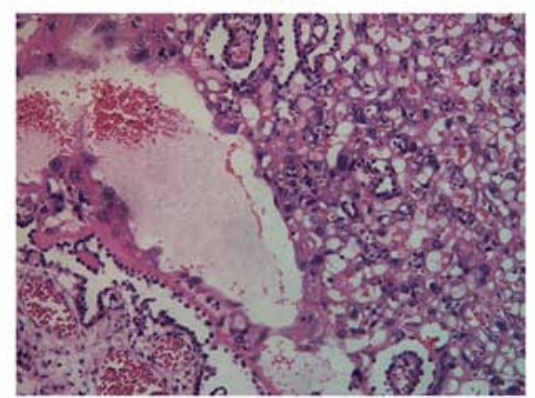

GDM+miR-875-5p inhibitor+Sh-TXNRD1

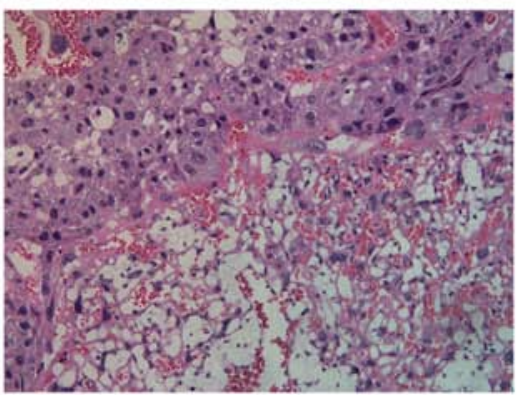

GDM+miR-875-5p inhibitor

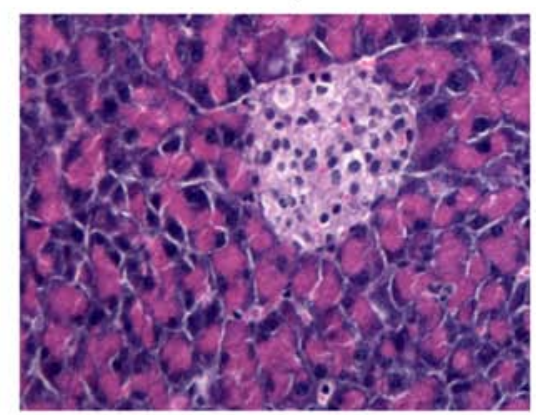

GDM+miR-875-5p inhibitor

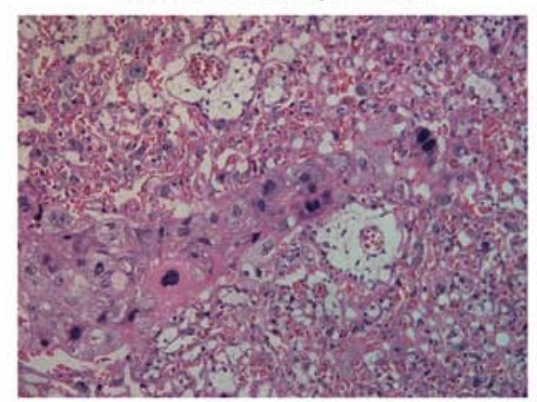

Figure 6. TXNRD1 knockdown reverses the effects of miR-875-5p inhibitor on pancreas and placenta injury. (A) Transfection with miR-875-5p inhibitor notably increased pancreatic injury. Arrows indicate islet cells. (B) Transfection with miR-875-5p inhibitor induced placental injury. Arrows indicate blood vessels and red blood cells. miR, microRNA; GDM, gestational diabetes mellitus; sh-, short hairpin RNA; TXNRD1, thioredoxin reductase 1 cytoplasmic; $\mathrm{NC}$, negative control.

significantly increased in GDM rats. The increase in TRXR1 mRNA levels induced by GDM may be caused by upregulation of TrxR enzyme activity, which can activate compensatory antioxidant defense systems of the body to protect cells from ROS-induced damage $(37,38)$. A previous study indicated that increased TrxR activity was positively associated with the upregulation of oxidative stress markers (39). The enhanced activity of the $\operatorname{Trx} / \operatorname{Trx} \mathrm{R}$ system is considered to play protective roles in preventing further complications of diabetes. Trx1 can reduce $\beta$-cell damage in non-obese diabetic subjects (40).
TrxR exerts anti-oxidative functions mainly by preventing stress-induced oxidative damage (29). Previous studies demonstrated that the transcription factor responsive element and the 3'UTR of TRXR1 could regulate its transcriptional activation or translation $(41,42)$. In addition, previous studies have shown that transcription factors, such as nuclear factor erythroid 2-related factor 2 and AP-1 regulate TRXR1 expression. In the present study, bioinformatic prediction and molecular biology experiments confirmed that miR-875-5p can specifically bind to TRXR1 3'UTR and inhibit the expression of endogenous 
TRXR1 at the post-transcriptional level to suppress its translation or induce degradation.

In conlcusion, the present study showed that miR-875-5p regulates IR and inflammation via targeting TXNRD1 in GDM model rats. Thus, these results indicated that miR-875-5p and TXNRD1 could be potential targets for treating GDM.

\section{Acknowledgements}

Not applicable.

\section{Funding}

The present study was supported by the Research Fund Project of the First Hospital of Lanzhou University (grant no. ldyyyn2018-59), the Research Fund Project of the Science and Technology Development Guiding Plan of Lanzhou City (grant no. 2019-ZD-38), Construction of Gansu Province Clinical Research Center for Endocrine disease (grant no. 20JR10FA667) and the Special Funds of Science and Technology Development of the Chinese Central Government to Guide Local in 2020 (an innovation platform for improving the prevention and treatment of frequently-occurring diseases in Gansu Province).

\section{Availability of data and materials}

The datasets used and/or analyzed during the current study are available from the corresponding author on reasonable request.

\section{Authors' contributions}

Songbo F made substantial contributions to the conception and design of the study, the acquisition, analysis and interpretation of data, the acquisition of funding, the collection of data and general supervision of the research group. Songquan F, XM, $\mathrm{XY}$ and JL made substantial contributions to the acquisition, analysis and interpretation of data. SongboF and SongquanF confirm the authenticity of all the raw data. All authors read and approved the final manuscript.

\section{Ethics approval and consent to participate}

All the experimental procedures were approved by the Animal Experimental Ethics Committee of The First Hospital of Lanzhou University.

\section{Patient consent for publication}

Not applicable.

\section{Competing interests}

The authors declare that they have no competing interests.

\section{References}

1. Metzger BE and Coustan DR; The Organizing Committee: Summary and recommendations of the Fourth International Workshop-Conference on Gestational Diabetes Mellitus. Diabetes Care 21 (Suppl 2): B161-B167, 1998.
2. Johns EC, Denison FC, Norman JE and Reynolds RM: Gestational Diabetes Mellitus: Mechanisms, Treatment, and Complications. Trends Endocrinol Metab 29: 743-754, 2018.

3. HajifarajiM,JahanjouF,AbbasalizadehF,AghamohammadzadehN, Abbasi MM and Dolatkhah N: Effect of probiotic supplements in women with gestational diabetes mellitus on inflammation and oxidative stress biomarkers: A randomized clinical trial. Asia Pac J Clin Nutr 27: 581-591, 2018

4. Sha H, Zeng H, Zhao J and Jin H: Mangiferin ameliorates gestational diabetes mellitus-induced placental oxidative stress, inflammation and endoplasmic reticulum stress and improves fetal outcomes in mice. Eur J Pharmacol 859: 172522, 2019.

5. Baig S, Rizi EP, Shabeer M and Agrawal M: Heredity of type 2 diabetes confers increased susceptibility to oxidative stress and inflammation. BMJ Open Diabetes Res Care 8: e000945, 2020.

6. Nazem MR, Asadi M, Jabbari N and Allameh A: Effects of zinc supplementation on superoxide dismutase activity and gene expression, and metabolic parameters in overweight type 2 diabetes patients: A randomized, double-blind, controlled trial. Clin Biochem 69: 15-20, 2019.

7. Sudharshana Murthy KA, Bhandiwada A, Chandan SL, Gowda SL and Sindhusree G: Evaluation of Oxidative Stress and Proinflammatory Cytokines in Gestational Diabetes Mellitus and Their Correlation with Pregnancy Outcome. Indian J Endocrinol Metab 22: 79-84, 2018.

8. Elliott HR, Sharp GC, Relton CL and Lawlor DA: Epigenetics and gestational diabetes: a review of epigenetic epidemiology studies and their use to explore epigenetic mediation and improve prediction. Diabetologia 62: 2171-2178, 2019.

9. Vasu S, Kumano K, Darden CM, Rahman I, Lawrence MC and Naziruddin B: MicroRNA Signatures as Future Biomarkers for Diagnosis of Diabetes States. Cells 8: E1533, 2019.

10. Zhao $\mathrm{H}$ and Tao S: miRNA-221 protects islet beta cell function in gestational diabetes mellitus by targeting PAK1. Biochem Biophys Res Commun 520: 218-224, 2008.

11. Zamanian Azodi M, Rezaei-Tavirani M, Rezaei-Tavirani M and Robati RM: Gestational Diabetes Mellitus Regulatory Network Identifies hsa-miR-145-5p and hsa-miR-875-5p as Potential Biomarkers. Int J Endocrinol Metab 17: e86640, 2019.

12. Xu Q, Zhu Q, Zhou Z, Wang Y, Liu X, Yin G, Tong X and Tu K: MicroRNA-876-5p inhibits epithelial-mesenchymal transition and metastasis of hepatocellular carcinoma by targeting BCL6 corepressor like 1. Biomed Pharmacother 103: 645-652, 2018.

13. Zhang T, Cai X, Li Q, Xue P, Chen Z, Dong X and Xue Y: Hsa-miR-875-5p exerts tumor suppressor function through down-regulation of EGFR in colorectal carcinoma (CRC). Oncotarget 7: 42225-42240, 2016.

14. Cebula M, Schmidt EE and Arnér ES: TrxR1 as a potent regulator of the Nrf2-Keap1 response system. Antioxid Redox Signal 23: 823-853, 2015.

15. Kabuyama Y, Kitamura T, Yamaki J, Homma MK, Kikuchi SI and Homma Y: Involvement of thioredoxin reductase 1 in the regulation of redox balance and viability of rheumatoid synovial cells. Biochem Biophys Res Commun 367: 491-496, 2008

16. Chang E, Kim DH, Yang H, Lee DH, Bae SH and Park CY: CB1 receptor blockade ameliorates hepatic fat infiltration and inflammation and increases Nrf2-AMPK pathway in a rat model of severely uncontrolled diabetes. PLoS One 13: e0206152, 2018.

17. Park HR, Lee SE, Yang H, Son GW, Jin YH and Park YS: Induction of Thioredoxin Reductase 1 by Korean Red Ginseng Water Extract Regulates Cytoprotective Effects on Human Endothelial Cells. Evid Based Complement Alternat Med 2015: 972040, 2015.

18. Raninga PV, Di Trapani G, Vuckovic S and Tonissen KF: TrxR1 inhibition overcomes both hypoxia-induced and acquired bortezomib resistance in multiple myeloma through NF-к $\beta$ inhibition. Cell Cycle 15: 559-572, 2016.

19. Matsui M, Oshima M, Oshima H, Takaku K, Maruyama T, Yodoi J and Taketo MM: Early embryonic lethality caused by targeted disruption of the mouse thioredoxin gene. Dev Biol 178: 179-185, 1996.

20. Shi X, Wang W, Zheng S, Zhang Q and Xu S: Selenomethionine relieves inflammation in the chicken trachea caused by LPS though inhibiting the NF- $\kappa$ B pathway. Biol Trace Elem Res 194: 525-535, 2020.

21. Ingram S, Mengozzi M, Heikal L, Mullen L and Ghezzi, P: Inflammation-induced reactive nitrogen species cause proteasomal degradation of dimeric peroxiredoxin- 1 in a mouse macrophage cell line. Free Radic Res 53: 875-881, 2019. 
22. John CM, Ramasamy R, Al Naqeeb G, Al-Nuaimi AH and Adam A: Nicotinamide supplementation protects gestational diabetic rats by reducing oxidative stress and enhancing immune responses. Curr Med Chem 19: 5181-5186, 2012.

23. Livak KJ, Schmittgen TD. Analysis of relative gene expression data using real-time quantitative PCR and the 2(-Delta Delta C(T)) Method. Methods 25: 402-408, 2001.

24. Bateman RM, Sharpe MD, Jagger JE, Ellis CG, Solé-Violán J, López-Rodríguez M, Herrera-Ramos E, Ruíz-Hernández J, Borderías L, Horcajada J, et al: 36th International Symposium on Intensive Care and Emergency Medicine: Brussels, Belgium. 15-18 March 2016. Crit Care 20: 94, 2016.

25. Kaushik SV, Plaisance EP, Kim T, Huang EY, Mahurin AJ, Grandjean PW and Mathews ST: Extended-release niacin decreases serum fetuin-A concentrations in individuals with metabolic syndrome. Diabetes Metab Res Rev 25: 427-434, 2009.

26. Skórzyńska-Dziduszko KE, Kimber-Trojnar Ż, Patro-Małysza J, Olszewska A, Zaborowski T and Małecka-Massalska T: An Interplay between Obesity and Inflammation in Gestational Diabetes Mellitus. Curr Pharm Biotechnol 17: 603-613, 2016

27. Roca-Rodríguez MDM, López-Tinoco C, Fernández-Deudero Á, Murri M, García-Palacios MV, García-Valero MDA, Tinahones FJ and Aguilar-Diosdado M: Unfavorable cytokine and adhesion molecule profiles during and after pregnancy, in women with gestational diabetes mellitus. Endocrinol Diabetes Nutr 64: 18-25, 2017.

28. Poston L, Caleyachetty R, Cnattingius S, Corvalán C, Uauy R, Herring $\mathrm{S}$ and Gillman MW: Preconceptional and maternal obesity: epidemiology and health consequences. Lancet Diabetes Endocrinol 12: 1025-1036, 2016

29. Tinkov AA, Bjørklund G, Skalny AV, Holmgren A, Skalnaya MG, Chirumbolo $S$ and Aaseth J: The role of the thioredoxin/thioredoxin reductase system in the metabolic syndrome: Towards a possible prognostic marker? Cell Mol Life Sci 75: 1567-1586, 2018.

30. Feng Y, Feng Q, Qu H, Song X, Hu J, Xu X, Zhang L and Yin S: Stress adaptation is associated with insulin resistance in women with gestational diabetes mellitus. Nutr Diab 10: 4, 2020.

31. Rueangdetnarong H, Sekararithi R, Jaiwongkam T, Kumfu S, Chattipakorn N, Tongsong T and Jatavan P: Comparisons of the oxidative stress biomarkers levels in gestational diabetes mellitus (GDM) and non-GDM among Thai population: cohort study. Endocrine Connect 7: 681-687, 2018.

32. Zygula A,Kosinski P,Zwierzchowska A,Sochacka M,WroczynskiP, Makarewicz-Wujec M, Pietrzak B, Wielgos M, Rzentala M and Giebultowicz J: Oxidative stress markers in saliva and plasma differ between diet-controlled and insulin-controlled gestational diabetes mellitus. Diabetes Res Clin Pract 148: 72-80, 2019.
33. Ren X, Zou L, Lu J and Holmgren A: Selenocysteine in mammalian thioredoxin reductase and application of ebselen as a therapeutic. Free Radic Biol Med 127: 238-247, 2018.

34. Lu J and Holmgren A: The thioredoxin antioxidant system. Free Radic Biol Med 66: 75-87, 2014.

35. Arnér ESJ: Focus on mammalian thioredoxin reductases Important selenoproteins with versatile functions. Biochim Biophys Acta 1790: 495-526, 2009.

36. Bobba A, Casalino E, Petragallo VA and Atlante A: Thioredoxin/thioredoxin reductase system involvement in cerebellar granule cell apoptosis. Apoptosis 19: 1497-1508, 2014.

37. Sun QA, Wu Y, Zappacosta F, Jeang KT, Lee BJ, Hatfield DL and Gladyshev VN: Redox Regulation of Cell Signaling by Selenocysteine in Mammalian Thioredoxin Reductases. J Biol Chem 274: 24522-24530, 1999.

38. He L, He T, Farrar S, Ji L, Liu T and Ma X: Antioxidants Maintain Cellular Redox Homeostasis by Elimination of Reactive Oxygen Species. Cell Physiol Biochem 44: 532-553, 2017.

39. Calabrese V, Mancuso C, Sapienza M, Puleo E, Calafato S, Cornelius C, Finocchiaro M, Mangiameli A, Di Mauro M, Stella AM and Castellino P: Oxidative stress and cellular stress response in diabetic nephropathy. Cell Stress Chaperones 12: 299-306, 2007.

40. Chernatynskaya AV, Looney B, Hu H, Zhu X and Xia CQ: Administration of recombinant human thioredoxin-1 significantly delays and prevents autoimmune diabetes in nonobese diabetic mice through modulation of autoimmunity. Diabetes Metab Res Rev 27: 809-812, 2011.

41. Gasdaska JR, Harney JW, Gasdaska PY, Powis G and Berry MJ: Regulation of Human Thioredoxin Reductase Expression and Activity by 3'-Untranslated Region Selenocysteine Insertion Sequence and mRNA Instability Elements. J Biol Chem 274: 25379-25385, 1999.

42. Rundlöf AK, Carlsten M and Arnér ESJ: The core promoter of human thioredoxin reductase 1: Cloning, transcriptional activity, and Oct-1, Sp1, and Sp3 binding reveal a housekeeping-type promoter for the AU-rich element-regulated gene. J Biol Chem 276: 30542-30551, 2001.

(i) $($ ) This work is licensed under a Creative Commons Attribution-NonCommercial-NoDerivatives 4.0 International (CC BY-NC-ND 4.0) License. 